J. Braz. Chem. Soc., Vol. 18, No. 1, 184-188, 2007.

Printed in Brazil - (C2007 Sociedade Brasileira de Química

0103 - $5053 \$ 6.00+0.00$

\title{
Phytochemical and Antibacterial Evaluation of Essential Oils from Ottonia Martiana Miq. (Piperaceae)
}

\author{
Miriam M. Cunico, ${ }^{a}$ André R. Lopes, ${ }^{b}$ Liliam C. Côcco, ${ }^{b}$ Carlos I. Yamamoto, ${ }^{b}$ Rubia C. B. Plocharski, ${ }^{b}$ \\ Marilis D. Miguel, ${ }^{a}$ Albino G. Junior, ${ }^{c}$ Celso G. Auer ${ }^{c}$ and Obdulio G. Miguel ${ }^{*, a}$ \\ ${ }^{a}$ Departamento de Farmácia, Universidade Federal do Paraná, Av. Prefeito Lothário Meissner, 632, \\ 80210-170 Curitiba-PR, Brazil \\ ${ }^{b}$ Laboratório de Análises de Combustíveis Automotivos (LACAUT), Centro Politécnico, Universidade Federal do \\ Paraná, Jardim das Américas, 81531-990 Curitiba-PR, Brazil
}

${ }^{c}$ Laboratório de Fitopatologia, Embrapa Florestas, Estrada da Ribeira, km 111, 83411-000 Colombo-PR, Brazil

Três óleos essenciais extraídos das folhas, frutos e raízes de Ottonia martiana Miq. (Piperaceae), espécie comum da floresta Atlântica brasileira, e conhecida popularmente por "anestésia", foram analisados por CG-EM e submetidos a um ensaio antibacteriano bioautográfico frente a Staphylococcus aureus (ATCC 25923), Staphylococcus epidermidis (ATCC 12228), Pseudomonas aerogenes (ATCC 27853) e Escherichia coli (ATCC 25922). Setenta e sete compostos foram identificados e submetidos a uma análise comparativa, a qual revelou uma variabilidade no teor dos componentes majoritários desses óleos (espatulenol, óxido de cariofileno, $(E)$-nerolidol, viridiflorol, $\beta$-cariofileno, $\delta$ cadineno e aloaromadendreno). A presença de zonas de inibição de crescimento bacteriano nos bioautogramas analisados ( $R f_{s} 0,29$ e 0,34) revelou o potencial antibacteriano dos óleos analisados frente às bactérias Gram-positivas testadas e permitiu identificar alguns dos componentes bioativos.

Three essential oils extracted from leaves, fruits and roots of Ottonia martiana Miq. (Piperaceae), common species in Brazilian Rain Forest, known as "anestésia", were analyzed by GC-MS and tested in an antibacterial assay against Staphylococcus aureus (ATCC 25923), Staphylococcus epidermidis (ATCC 12228), Pseudomonas aerogenes (ATCC 27853) and Escherichia coli (ATCC 25922). Seventy-seven compounds were identified and submitted to a comparative analysis, which revealed variability on the amount of principal components of these oils (spathulenol, caryophyllene oxide, $(E)$-nerolidol, viridiflorol, $\beta$-caryophyllene, $\delta$ cadinene and alloaromadendrene). Inhibition zones of bacterial growth in the bioautograms (Rfs 0.29 and 0.34 ) showed antimicrobial activity of essential oils against tested Gram-positive bacteria and permitted to identify some bioactive components.

Keywords: antimicrobial activity, essential oil composition, Gram-positive bacteria, Ottonia martiana

\section{Introduction}

Actually, research of healthy habits for world population has promoted a progressive increase on production and consumption of natural products, as the case of essential oils from plants, source of bioactive substances. ${ }^{1}$

Chemical investigation of Piperaceae essential oils has revealed the presence of monoterpenes, sesquiterpenes and arylpropanoids that have shown interesting biological

*e-mail: obdulio@ufpr.br properties including psychotropic, antimicrobial, antioxidant and cytotoxic effects stimulating studies on plants within this family. ${ }^{2}$

Among Piperaceae species of medicinal interest, there is Ottonia martiana Miq, a characteristic shrub of the Brazilian Rain Forest ${ }^{3,4}$ and known as "anestésia" for the natives of the Paraná coast due to its anesthetically action on the oral mucous. ${ }^{5,6}$

Phytochemical investigations with roots and aerial parts of "anestésia" revealed some isobutylamides (piperlonguminine, isopiperlonguminine and piperovatine), compounds of notable biological properties. ${ }^{7-9}$ 
No chemical data on essential oil of $O$. martiana has been found, but two papers discussing on essential oils constitution of other species from genus Ottonia have been previously published. ${ }^{10,11}$

This work relates a phytochemical investigation on essential oils extracted from leaves, fruits and roots of $O$. martiana and their antibacterial activity.

\section{Experimental}

\section{General}

All reagents were of analytical grade. Column chromatography and TLC were performed on silica gel $60 \mathrm{~F}_{254}$ (Merck). Mass spectra was determined with a Varian 3800 Series GC-EIMS (Varian SATURN 2000), $70 \mathrm{eV}$, capillary column CP-SIL PONA CB $(100 \mathrm{~m} \times 0.25$ mm I.D. $\times 0.25 \mu \mathrm{m}$ film thickness) with helium as the carrier gas at a flow rate of $1.6 \mathrm{~mL} \mathrm{~min}^{-1}$; the temperature program was $140{ }^{\circ} \mathrm{C}(10 \mathrm{~min})$ and increased at a rate of $5^{\circ} \mathrm{C}$ min $^{-1}$ to $230{ }^{\circ} \mathrm{C}$, standing $25 \mathrm{~min}$ at this temperature; injection in the split mode $(1: 200)$ at an injector, temperature of $200{ }^{\circ} \mathrm{C}$; detector temperature at $200{ }^{\circ} \mathrm{C}$; injection volume, $0.5 \mu \mathrm{L}$. Individual components were identified by comparison of both mass spectrum and their GC retention times (RT) and retention indices (RI) with those of authentic compounds previously analyzed and stored in the data system or existing in the literature. ${ }^{10-12}$ The NIST (National Institute for Standard Technology 62.235 compounds) was used for comparison of mass spectra. The retention indices were calculated for all volatile constituents using a homologous series $\left(\mathrm{C}_{9}\right.$ to $\left.\mathrm{C}_{20}\right)$ recorded under the same operating conditions. Retention indices (RI) have been obtained according to the method of Van den Dool. ${ }^{13}$ The quantitative data were obtained by electronic integration of the GC-FID peak areas.

The bacterial strains were provided by the Laboratory of Basic Pathology-Universidade Federal do Paraná. Tests were carried out in duplicate with strains of Staphylococcus aureus (ATCC 25923), Staphylococcus epidermidis (ATCC 12228), Pseudomonas aerogenes (ATCC 27853) and Escherichia coli (ATCC 25922). New cultures of each strain were standardized in sterile saline solution following 0.5 MacFarland scale. Miller Hinton Agar (Difco Laboratories) was used for culture medium.

\section{Plant material}

Roots and aerial parts (leaves and fruits) of $O$. martiana were collected in February 2003, in Paraná State, Southern region of Brazil. The botanist Dr. Gerdt Hatschbach of the Museu Botânico Municipal - Prefeitura de Curitiba, Paraná (MBM) identified the plant. Voucher specimens are deposited at the Herbarium of the MBM under number 259057.

\section{Extraction of the oils}

Essential oils of leaves (188.4 g) and fruits (30.0 g) from $O$. martiana were obtained by hydrodistillation for four hours in a modified Clevenger-type apparatus, after cooling to $0{ }^{\circ} \mathrm{C}$.

The essential oil of roots was obtained in previously phytochemical investigation. The air-dried and powdered roots of $O$. martiana $(470.0 \mathrm{~g})$ were extracted with $95 \%$ ethanol in a Soxhlet for $5 \mathrm{~h}$ at hot stage $(470 \mathrm{~g} / 3 \mathrm{~L})$. The obtained filtered material, after removal of the ethanol under reduced pressure was partitioned with $250 \mathrm{~mL}$ of hexane, dichloromethane, ethyl acetate and methanol, respectively. The fraction hexane $(4.0 \mathrm{~g})$ obtained after evaporation of the solvent under vacuum at $40{ }^{\circ} \mathrm{C}$, was submitted to column cromatography on a column of silica gel and developed with solvent mixtures of increasing polarity at 5\% (hexane, hexane/ethyl acetate, ethyl acetate/ methanol and methanol), yielding $0.1 \mathrm{~mL}$ of the colorless essential oil $(0.02 \%)$ correspondent to the grouped subfractions 5-13.

\section{Antimicrobial activity assay}

The bioautography method, ${ }^{14}$ adapted from Hostettmann ${ }^{15}$ was based on the application of $3 \mu \mathrm{L}$ of the test oils on a TLC plate. The samples were first applied on a TLC plate $\mathrm{GF}_{254}(2.5 \times 5.0 \mathrm{~cm})$ and developed with toluene/EtOAc (97:3), transferred to a Petri dish, covered with Miller Hinton Agar containing triphenyltetrazolium chloride (TTC - 1\%) and inoculated with bacterial strains previously standardized. Inhibition zones after $24 \mathrm{~h}$ of incubation $\left(37^{\circ} \mathrm{C}\right)$ indicated the presence of active compounds. Chloramphenicol (Newprov - $3 \mu \mathrm{L}$ ) was used as positive control of growth inhibition.

MIC tests were carried out according to Eloff, ${ }^{16}$ using a tissue culture testplate (96 wells). The stock solutions of the oils were diluted and transferred into the first well $(100 \mu \mathrm{L})$, and serial dilutions were performed so that concentrations in the range of $1-0.001 \mathrm{mg} \mathrm{mL}^{-1}$ were obtained. Chloramphenicol (Merck) was used as the reference antibiotic control. The inoculum was added to all wells and the plates were incubated at $37{ }^{\circ} \mathrm{C}$ during $24 \mathrm{~h}$. Antimicrobial activity was detected by adding $20 \mu \mathrm{L}$ of $1 \%$ TTC aqueous solution. MIC was defined as the lowest concentration of oil that inhibited visible growth. 


\section{Results and Discussion}

The oils were obtained from dried plant material yielding $0.21 \%$ (leaves), $0.33 \%$ (fruits) and $0.02 \%$ (roots).

A total of 77 compounds were identified, accounting for 68.22 to $78.87 \%$ of the constituents. The identification of the constituents was performed by computer library search, retention indices (RI) and visual interpretation of the mass spectra. ${ }^{12}$ Results obtained for the qualitative and quantitative analysis of these essential oils are shown in Table 1.
The three oils showed some differences in their main constituents. The major components of root oil were spathulenol (17.83\%), (E)-nerolidol (10.33\%), viridiflorol (7.53\%), caryophyllene oxide $(6.41 \%), \delta$-cadinene (4.16\%), myrcene (4.07\%), $\alpha$-copaene (3.86\%), $\beta$ caryophyllene $(3.21 \%)$, allaromadendrene $(3.11 \%)$ and $\alpha$-pinene $(2.10 \%)$. The fruit oil was characterized by its high content of spathulenol $(17.37 \%),(E)$-nerolidol $(9.14 \%)$, viridiflorol $(7.38 \%)$, caryophyllene oxide $(5.77 \%), \delta$-cadinene $(3.90 \%)$, myrcene $(3.84 \%)$, $\alpha$-copaene (3.69\%), $\beta$-caryophyllene (3.07\%), alloaroma-

Table 1. Essential oils composition from Ottonia martiana Miq., Piperaceae

\begin{tabular}{|c|c|c|c|c|c|c|c|c|}
\hline & Compounds & Formula & MW & $\mathrm{Rt} / \mathrm{min}$ & RI & Leaves/(\%) & Fruits/(\%) & Roots/(\%) \\
\hline 01 & $\alpha$-Thujene & $\mathrm{C}_{10} \mathrm{H}_{16}$ & 136 & 13.205 & 929 & $<0.01$ & 0.02 & 0.02 \\
\hline 02 & $\alpha$-Pinene & $\mathrm{C}_{10} \mathrm{H}_{16}$ & 136 & 13.703 & 941 & 1.30 & 2.01 & 2.10 \\
\hline 03 & 6-Methyl-5-hepten-2-one & $\mathrm{C}_{8} \mathrm{H}_{14} \mathrm{O}$ & 126 & 14.431 & 958 & 0.02 & 0.54 & 0.56 \\
\hline 04 & Camphene & $\mathrm{C}_{10}^{0} \mathrm{H}_{16}$ & 136 & 14.448 & 958 & 0.02 & 0.04 & 0.05 \\
\hline 05 & Sabinene & $\mathrm{C}_{10} \mathrm{H}_{16}$ & 136 & 15.174 & 975 & 0.50 & $<0.01$ & $<0.01$ \\
\hline 06 & Myrcene & $\mathrm{C}_{10} \mathrm{H}_{16}$ & 136 & 15.407 & 981 & 1.01 & 3.84 & 4.07 \\
\hline 07 & $\beta$-Pinene & $\mathrm{C}_{10} \mathrm{H}_{16}$ & 136 & 15.656 & 987 & 0.37 & 0.72 & 0.76 \\
\hline 08 & $\alpha$-Phellandrene & $\mathrm{C}_{10} \mathrm{H}_{16}$ & 136 & 16.734 & 1007 & 0.01 & $<0.01$ & $<0.01$ \\
\hline 09 & $\delta$-3-Carene & $\mathrm{C}_{10} \mathrm{H}_{16}$ & 136 & 17.271 & 1015 & $<0.01$ & 0.03 & 0.03 \\
\hline 10 & $\alpha$-Terpinene & $\mathrm{C}_{10} \mathrm{H}_{16}$ & 136 & 17.420 & 1017 & $<0.01$ & $<0.01$ & $<0.01$ \\
\hline 11 & $p$-Cymene & $\mathrm{C}_{10} \mathrm{H}_{14}$ & 134 & 17.537 & 1018 & 0.22 & 0.42 & 0.45 \\
\hline 12 & Limonene & $\mathrm{C}_{10} \mathrm{H}_{16}$ & 136 & 18.203 & 1028 & 0.37 & 0.59 & 0.63 \\
\hline 13 & $\beta$-phellandrene & $\mathrm{C}_{10} \mathrm{H}_{16}$ & 136 & 18.335 & 1030 & 0.04 & 0.08 & 0.09 \\
\hline 14 & cis-Ocimene & $\mathrm{C}_{10} \mathrm{H}_{16}$ & 136 & 18.655 & 1034 & $<0.01$ & 0.02 & 0.02 \\
\hline 15 & $\gamma$-Terpinene & $\mathrm{C}_{10} \mathrm{H}_{16}$ & 136 & 20.021 & 1053 & 0.01 & 0.02 & 0.02 \\
\hline 16 & (Z)-Linalool oxide & $\mathrm{C}_{10} \mathrm{H}_{18} \mathrm{O}_{2}$ & 170 & 20.791 & 1064 & $<0.01$ & 0.21 & 0.21 \\
\hline 17 & p-Cymenene & $\mathrm{C}_{10} \mathrm{H}_{22}$ & 132 & 22.000 & 1081 & $<0.01$ & 0.03 & 0.03 \\
\hline 18 & Linalool & $\mathrm{C}_{10} \mathrm{H}_{18} \mathrm{O}$ & 154 & 22.379 & 1086 & 0.06 & 0.19 & 0.19 \\
\hline 19 & $\alpha$-Campholenal & $\mathrm{C}_{10} \mathrm{H}_{16} \mathrm{O}$ & 152 & 24.471 & 1120 & $<0.01$ & 0.20 & 0.21 \\
\hline 20 & trans-Pinocarveol & $\mathrm{C}_{10} \mathrm{H}_{16} \mathrm{O}$ & 152 & 25.778 & 1143 & 0.02 & 0.14 & 0.14 \\
\hline 21 & trans-Verbenol & $\mathrm{C}_{10} \mathrm{H}_{16} \mathrm{O}$ & 152 & 25.832 & 1144 & 0.01 & 0.13 & 0.13 \\
\hline 22 & Pinocarvone & $\mathrm{C}_{10} \mathrm{H}_{14} \mathrm{O}$ & 150 & 26.620 & 1158 & $<0.01$ & 0.03 & 0.03 \\
\hline 23 & p-Cymen-8-ol & $\mathrm{C}_{10} \mathrm{H}_{14} \mathrm{O}$ & 150 & 27.342 & 1172 & $<0.01$ & 0.01 & 0.01 \\
\hline 24 & Terpinen-4-ol & $\mathrm{C}_{10} \mathrm{H}_{18} \mathrm{O}$ & 154 & 27.643 & 1177 & $<0.01$ & 0.08 & 0.02 \\
\hline 25 & $\alpha$-Terpineol & $\mathrm{C}_{10} \mathrm{H}_{18} \mathrm{O}$ & 154 & 28.085 & 1185 & $<0.01$ & 0.03 & 0.05 \\
\hline 26 & Myrtenal & $\mathrm{C}_{10} \mathrm{H}_{14} \mathrm{O}$ & 150 & 28.176 & 1187 & 0.03 & 0.02 & 0.03 \\
\hline 27 & Myrtenol & $\mathrm{C}_{10} \mathrm{H}_{16} \mathrm{O}$ & 152 & 28.423 & 1191 & 0.02 & 0.03 & 0.04 \\
\hline 28 & Verbenone & $\mathrm{C}_{10} \mathrm{H}_{14} \mathrm{O}$ & 150 & 28.718 & 1196 & $<0.01$ & 0.05 & 0.06 \\
\hline 29 & trans-Carveol & $\mathrm{C}_{10} \mathrm{H}_{16} \mathrm{O}$ & 152 & 29.150 & 1206 & $<0.01$ & 0.03 & 0.05 \\
\hline 30 & Nerol (cis-geraniol) & $\mathrm{C}_{10} \mathrm{H}_{18} \mathrm{O}$ & 154 & 29.508 & 1215 & 0.01 & 0.04 & 0.06 \\
\hline 31 & $p$-Anisaldehyde & $\mathrm{C}_{8} \mathrm{H}_{8} \mathrm{O}_{2}$ & 136 & 29.961 & 1227 & $<0.01$ & 0.06 & 0.02 \\
\hline 32 & Carvone & $\mathrm{C}_{10} \mathrm{H}_{14} \mathrm{O}$ & 150 & 30.066 & 1229 & 0.01 & 0.05 & 0.01 \\
\hline 33 & trans-Geraniol & $\mathrm{C}_{10} \mathrm{H}_{18} \mathrm{O}$ & 154 & 30.424 & 1238 & 0.05 & 0.06 & 0.07 \\
\hline 34 & Piperitone & $\mathrm{C}_{10} \mathrm{H}_{16} \mathrm{O}$ & 152 & 30.615 & 1243 & 0.06 & 0.02 & 0.05 \\
\hline 35 & Geranial (a-citral) & $\mathrm{C}_{10} \mathrm{H}_{16} \mathrm{O}$ & 152 & 30.829 & 1249 & $<0.01$ & 0.01 & 0.01 \\
\hline 36 & $N$-decanol & $\mathrm{C}_{16} \mathrm{H}_{32} \mathrm{O}$ & 256 & 31.093 & 1256 & $<0.01$ & 0.07 & 0.01 \\
\hline 37 & $\alpha$-Terpinen-7-al & $\mathrm{C}_{10} \mathrm{H}_{14} \mathrm{O}$ & 150 & 31.307 & 1261 & 0.06 & 0.50 & 0.01 \\
\hline 38 & cis-Verbenyl acetate & $\mathrm{C}_{12} \mathrm{H}_{18} \mathrm{O}_{2}$ & 194 & 31.425 & 1264 & $<0.01$ & 0.01 & 0.01 \\
\hline 39 & Indole & $\mathrm{C}_{8} \mathrm{H}_{7} \mathrm{~N}$ & 117 & 31.471 & 1265 & $<0.01$ & 0.01 & 0.01 \\
\hline 40 & p-Cymen-7-ol & $\mathrm{C}_{10} \mathrm{H}_{14} \mathrm{O}$ & 150 & 31.716 & 1271 & 0.06 & 0.01 & 0.02 \\
\hline 41 & Tymol & $\mathrm{C}_{10} \mathrm{H}_{14} \mathrm{O}$ & 150 & 32.012 & 1279 & 0.07 & 0.01 & $<0.01$ \\
\hline 42 & Perilla alcohol & $\mathrm{C}_{10} \mathrm{H}_{16} \mathrm{O}$ & 152 & 32.329 & 1287 & $<0.01$ & 0.01 & $<0.01$ \\
\hline 43 & $N$-tridecane & $\mathrm{C}_{13} \mathrm{H}_{28}$ & 184 & 32.861 & 1301 & 0.07 & 0.02 & $<0.01$ \\
\hline 44 & Piperonal & $\mathrm{C}_{8} \mathrm{H}_{6} \mathrm{O}_{3}$ & 150 & 33.000 & 1305 & $<0.01$ & 0.03 & $<0.01$ \\
\hline 45 & $\delta$-Elemene & $\mathrm{C}_{15} \mathrm{H}_{24}$ & 204 & 34.439 & 1351 & $<0.01$ & 0.60 & 0.10 \\
\hline 46 & $\alpha$-Cubebene & $\mathrm{C}_{15} \mathrm{H}_{24}$ & 204 & 34.886 & 1366 & 0.48 & 0.28 & 0.30 \\
\hline 47 & Isoledene & $\mathrm{C}_{15} \mathrm{H}_{24}$ & 204 & 35.593 & 1388 & $<0.01$ & $<0.01$ & $<0.01$ \\
\hline
\end{tabular}


Table 1. cont.

\begin{tabular}{|c|c|c|c|c|c|c|c|c|}
\hline & Compounds & Formula & MW & $\mathrm{Rt} / \mathrm{min}$ & RI & Leaves/(\%) & Fruits/(\%) & Roots/(\%) \\
\hline 48 & $\alpha$-Ylangene & $\mathrm{C}_{15} \mathrm{H}_{24}$ & 204 & 35.714 & 1392 & $<0.01$ & $<0.01$ & $<0.01$ \\
\hline 49 & Cyclosativene & $\mathrm{C}_{15} \mathrm{H}_{24}$ & 204 & 35.782 & 1394 & $<0.01$ & 0.03 & 0.04 \\
\hline 50 & $\alpha$-Copaene & $\mathrm{C}_{15} \mathrm{H}_{24}$ & 204 & 35.858 & 1397 & 4.41 & 3.69 & 3.86 \\
\hline 51 & $\beta$-Elemene & $\mathrm{C}_{15} \mathrm{H}_{24}$ & 204 & 36.108 & 1405 & 0.95 & 1.28 & 1.35 \\
\hline 52 & $\beta$-Bourbonene & $\mathrm{C}_{15} \mathrm{H}_{24}$ & 204 & 36.185 & 1408 & $<0.01$ & 0.18 & 0.18 \\
\hline 53 & Longifolene & $\mathrm{C}_{15} \mathrm{H}_{24}$ & 204 & 36.854 & 1432 & 0.12 & 0.03 & 0.09 \\
\hline 54 & $\alpha$-Gurjunene & $\mathrm{C}_{15} \mathrm{H}_{24}^{24}$ & 204 & 36.977 & 1436 & 0.59 & 0.41 & 0.43 \\
\hline 55 & $\beta$-Caryophyllene & $\mathrm{C}_{15} \mathrm{H}_{24}^{24}$ & 204 & 37.276 & 1447 & 12.61 & 3.07 & 3.21 \\
\hline 56 & epi-bicyclosesquiphellandrene & $\mathrm{C}_{15} \mathrm{H}_{24}$ & 204 & 37.468 & 1454 & 0.88 & 0.35 & 0.35 \\
\hline 57 & $\beta$-Gurjunene & $\mathrm{C}_{15} \mathrm{H}_{24}$ & 204 & 37.732 & 1463 & 1.49 & 0.79 & 0.83 \\
\hline 58 & Aromadendrene & $\mathrm{C}_{15} \mathrm{H}_{24}$ & 204 & 37.880 & 1468 & 0.14 & 0.11 & 0.12 \\
\hline 59 & $\alpha$-Humulene & $\mathrm{C}_{15} \mathrm{H}_{24}$ & 204 & 38.250 & 1482 & 0.31 & 0.57 & 0.76 \\
\hline 60 & Alloaromadendrene & $\mathrm{C}_{15} \mathrm{H}_{24}$ & 204 & 38.475 & 1490 & 3.15 & 2.97 & 3.11 \\
\hline 61 & $\gamma$-Muurolene & $\mathrm{C}_{15} \mathrm{H}_{24}$ & 204 & 38.596 & 1494 & 2.51 & 0.32 & 0.34 \\
\hline 62 & Germacrene D & $\mathrm{C}_{15} \mathrm{H}_{24}^{24}$ & 204 & 38.815 & 1501 & 0.19 & 0.47 & 0.49 \\
\hline 63 & $\gamma$-Cadinene & $\mathrm{C}_{15} \mathrm{H}_{24}$ & 204 & 38.915 & 1505 & 0.21 & 0.59 & 0.62 \\
\hline 64 & Valencene & $\mathrm{C}_{15} \mathrm{H}_{24}$ & 204 & 39.197 & 1515 & 0.21 & 0.15 & 0.15 \\
\hline 65 & epi- Cubebol & $\mathrm{C}_{15} \mathrm{H}_{26}{ }_{26} \mathrm{O}$ & 222 & 39.230 & 1517 & 0.33 & 1.17 & 1.21 \\
\hline 66 & Viridiflorene (ledene) & $\mathrm{C}_{15} \mathrm{H}_{24}$ & 204 & 39.316 & 1520 & 0.38 & 0.22 & 0.27 \\
\hline 67 & $\alpha$-Selinene & $\mathrm{C}_{15} \mathrm{H}_{24}$ & 204 & 39.388 & 1523 & 0.83 & 0.46 & 0.47 \\
\hline 68 & cis-Calamenene & $\mathrm{C}_{15} \mathrm{H}_{22}$ & 202 & 39.705 & 1534 & 0.10 & 0.12 & 0.13 \\
\hline 69 & $\delta$-Cadinene & $\mathrm{C}_{15} \mathrm{H}_{24}$ & 204 & 39.777 & 1537 & 3.74 & 3.90 & 4.16 \\
\hline 70 & $(E)$-Nerolidol & $\mathrm{C}_{15} \mathrm{H}_{26} \mathrm{O}$ & 222 & 40.278 & 1555 & 8.18 & 9.14 & 10.33 \\
\hline 71 & Elemol & $\mathrm{C}_{15} \mathrm{H}_{26} \mathrm{O}$ & 222 & 40.396 & 1559 & 0.48 & 0.82 & 0.85 \\
\hline 72 & Ledol & $\mathrm{C}_{15} \mathrm{H}_{26} \mathrm{O}$ & 222 & 41.425 & 1597 & 0.19 & 0.76 & 0.70 \\
\hline 73 & Spathulenol & $\mathrm{C}_{15} \mathrm{H}_{24} \mathrm{O}$ & 220 & 41.609 & 1603 & 8.59 & 17.37 & 17.83 \\
\hline 74 & Globulol & $\mathrm{C}_{15} \mathrm{H}_{26} \mathrm{O}^{24}$ & 222 & 41.813 & 1610 & 0.44 & 0.44 & 0.43 \\
\hline 75 & Caryophyllene oxide & $\mathrm{C}_{15} \mathrm{H}_{24} \mathrm{O}$ & 220 & 41.949 & 1614 & 7.37 & 5.77 & 6.41 \\
\hline 76 & Viridiflorol & $\mathrm{C}_{15} \mathrm{H}_{26} \mathrm{O}$ & 222 & 42.637 & 1636 & 2.75 & 7.38 & 7.53 \\
\hline \multirow[t]{2}{*}{77} & Cubenol & $\mathrm{C}_{15} \mathrm{H}_{26} \mathrm{O}$ & 222 & 43.104 & 1651 & 2.19 & 2.35 & 1.94 \\
\hline & Total identified & & & & & 68.22 & 75.77 & 78.87 \\
\hline
\end{tabular}

The identified contituents are listed in their order of elution from a nonpolar column (CP-SIL PONA CB); Rt - retention times; RI - retention indices (experimental data).

dendrene (2.97\%), cubenol (2.35\%) and $\alpha$-pinene (2.01\%). The most important constituents in leaf oil were $\beta$-caryophyllene (12.61\%), spathulenol (8.59\%), $(E)$ nerolidol $(8.18 \%)$, caryophyllene oxide $(7.37 \%), \alpha$ copaene $(4.41 \%), \delta$-cadinene $(3.74 \%)$, alloaromadendrene
(3.15\%), viridiflorol $(2.75 \%)$ and $\gamma$-muurolene $(2.51 \%)$. Several compounds of the oils remained unidentified.

The comparative analysis between oils showed variability in chemical constitution related to content of their principal components in different organs (Figure 1).

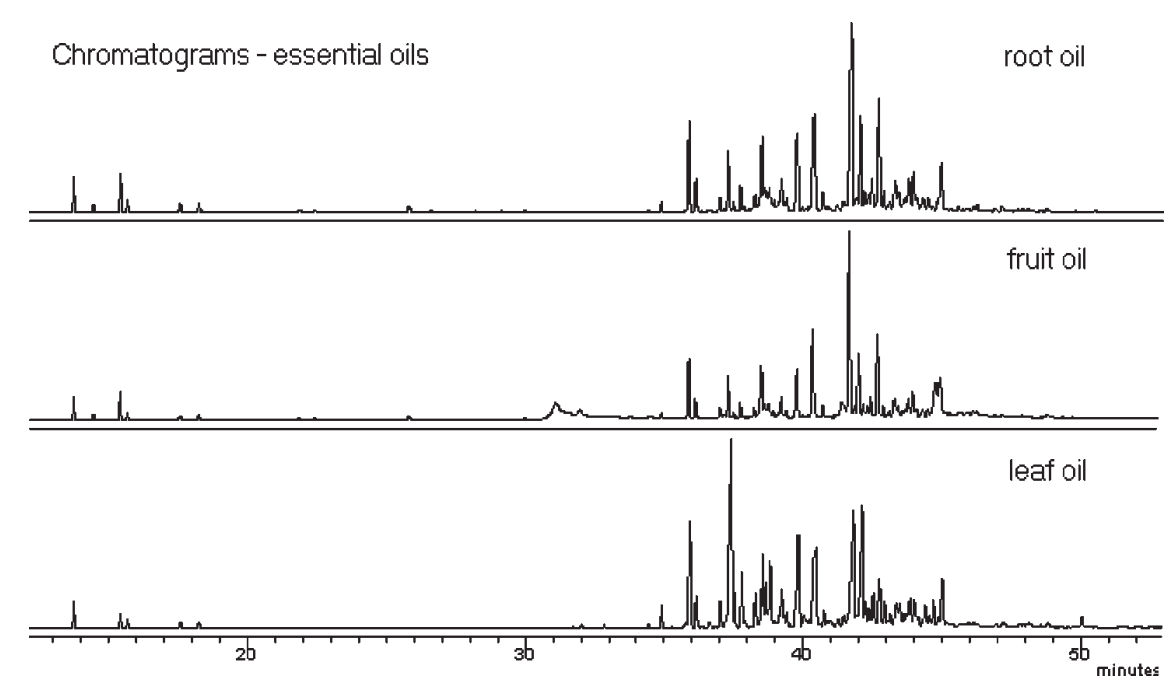

Figure 1. Chromatograms of GC-MS analysis of essential oils from the Ottonia martiana. 
Table 2. Antibacterial activity of essential oils from Ottonia martiana on four bacterial species

\begin{tabular}{|c|c|c|c|c|c|c|c|}
\hline \multirow{3}{*}{ Microrganisms(Bacteria) } & \multicolumn{7}{|c|}{ Inhibition zones (IZ) (Bioautography) } \\
\hline & \multirow[t]{2}{*}{ Gram } & \multicolumn{2}{|c|}{ leaves $3 \mu \mathrm{L}$} & \multicolumn{2}{|c|}{ fruits $3 \mu \mathrm{L}$} & \multicolumn{2}{|c|}{ roots $3 \mu \mathrm{L}$} \\
\hline & & IZ & $\mathrm{R} f s$ & $\mathrm{IZ}$ & $\mathrm{R} f s$ & $\mathrm{IZ}$ & $\mathrm{R} f s$ \\
\hline Staphylococcus aureus (ATCC 25923)) & Positive & ++ & 0.29 and 0.34 & ++ & 0.29 and 0.34 & ++ & 0.29 and 0.34 \\
\hline Staphylococcus epidermidis (ATCC 12228) & Positive & ++ & 0.29 and 0.34 & ++ & 0.29 and 0.34 & ++ & 0.29 and 0.34 \\
\hline Pseudomonas aerogenes (ATCC 27853) & Negative & - & - & - & - & - & - \\
\hline Escherichia coli (ATCC 25922) & Negative & - & - & - & - & - & - \\
\hline
\end{tabular}

ATCC (American Type Culture Collection); + = inhibition zone; - = no inhibition zone.

Compounds present in oils indicated a preference of Ottonia to synthesize sesquiterpenes and the commonest have the $E, E$-farnesyl-PP as fundamental precursor. ${ }^{10}$ Many described compounds as components of essential oils of $O$. anisum and $O$. corcovadensis ${ }^{10,11}$ also were detected during analysis of this research. This is the first report over a detailed composition of the essential oil present in roots, leaves and fruits of $O$. martiana.

Bioautography assay permitted to detect and identify bioactive compounds of $O$. martiana against all Grampositive bacteria tested (Table 2).

The bioautography method directioned isolation of the bioactive compounds towards their identification as $\alpha$-pinene ( $\mathrm{R} f 0.29), \beta$-pinene ( $\mathrm{R} f 0.29)$, myrcene ( $\mathrm{R} f 0.29)$, $\alpha$-copaene (Rf 0.34), $\beta$-caryophyllene ( $\mathrm{R} f 0.34)$ and $\delta$ cadinene ( $\mathrm{R} f 0.34$ ). Some of these compounds are previously known for its antimicrobial activity. ${ }^{17,18}$ The relative composition of each inhibition zone was characterised using GC-MS after preparative TLC. The inhibition zone with $\mathrm{R} f 0.34$ also contained smaller quantities of $\alpha$-cubebene, $\beta$-elemene, valencene, $\alpha$-gurjunene, epi-bicyclosesquiphellandrene and germacrene $\mathrm{D}$.

The MIC (Minimal Inhibitory Concentration) was determined for oils that presented positive results on bioautographic assays. The oils showed an activity against S. aureus with the MIC of $9 \mu \mathrm{g} \mathrm{mL}^{-1}$ (leaf oil), $5 \mu \mathrm{g} \mathrm{mL}^{-1}$ (fruit oil) and $5 \mu \mathrm{g} \mathrm{mL}^{-1}$ (root oil). This oils also showed an activity against $S$. epidermidis with the MIC of $9 \mu \mathrm{g}$ $\mathrm{mL}^{-1}$ (leaf oil), $5 \mu \mathrm{g} \mathrm{mL}^{-1}$ (fruit oil) and $5 \mu \mathrm{g} \mathrm{mL}^{-1}$ (root oil).

These results indicated an effective in vitro activity of the essential oils from $O$. martiana and encourage further studies for its application in antibiotic therapy of infectious diseases.

\section{References}

1. Kelsey, R. G.; Reynolds, G. W.; Rodriguez, E. In Biology and Chemistry of Plant Trichomes; Rodriguez, E.; Healey, P. L.; Mehta, I., eds.; Plenum Press: New York, 1984.
2. Moreira, D. L.; Souza, P. O.; Kaplan, M. A. C.; Pereira, N. A.; Cardoso, G. L.; Guimarães, E. F.; An. Acad. Bras.Cienc. 2001, $73,33$.

3. Yuncker, T. C.; Hoehnea 1973, 2, 131.

4. Franklin Guimarães, E.; Falcão Ichaso, C. L.; Gonçalves Costa, C.; Flora Ilustrada Catarinense, 1978, I, 1.

5. Lopes, M.; MSc. Dissertation, Universidade Federal do Paraná, Brazil, 1989.

6. Cunico, M. M.; Miguel, O. G.; Miguel, M. D.; Carvalho, J. L. S.; Montrucchio, D. P.; Ferreira, J. L; Oliveira, J. S.; Quim. Nova. 2003, 26, 803.

7. Cunico, M. M.; Miguel, O. G.; Miguel, M. D.; Kerber, V. A.; Montrucchio, D. P.; Auer, C. G.; Grigoletti Júnior, A.; Rev. Ciênc. Farm. 2003, 24, 141.

8. Cunico, M. M.; Carvalho, J. L. S.; Kerber, V. A.; Higaskino, C. E. K.; Cruz Almeida, S. C.; Miguel, M. D.; Miguel, O. G.; Rev. Bras. Farmacogn. 2004, 14, 97.

9. Cunico, M. M.; Miguel, O. G.; Miguel, M. D.; Carvalho, J. L. S.; Peitz, C.; Auer, C. G.; Grigoletti Júnior, A.; Revista Visão Acadêmica 2003, 4, 77.

10. Santos, P. R. D.; Moreira, D. L.; Guimarães, E. F.; Kaplan, M. A. C.; Phytochemistry 2001, 58, 547.

11. Facundo, V. A; Morais, S. M.; Braz Filho, R.; Quim. Nova, 2004, 27, 79.

12. Adams, R. P. Identification of Essential Oils by Gas Chromatography/Mass Spectroscopy, Allured Publishing Corporation: Carol Stream, IL, USA, 1995.

13. Van den Dool, H.; Kratz, P. D. A.; J. Chromatogr. 1963, 11, 463.

14. Weltzien, H. C.; Naturwissenschaften 1958, 45, 288.

15. http://www.iupac.org/symposia/proceedings/phuket97/ hostettmann.html, accessed in January 2005.

16. Eloff, J. N. P.; Planta Med. 1998, 64, 711.

17. Kang, R.; Helms, R.; Stout, M. J.; Jaber, H.; Nakatsu, T.; J. Agric. Food Chem. 1992, 40, 2328.

18. Kubo, I.; Muroi, H.; J. Agric. Food Chem. 1993, 41, 1102. 